\title{
New version of fractional Simpson type inequalities for twice differentiable functions
}

\author{
Fatih Hezenci ${ }^{1 *}$ (D, Hüseyin Budak ${ }^{1}$ and Hasan Kara
}

"Correspondence:

fatihezenci@gmail.com

'Department of Mathematics,

Faculty of Science and Arts, Düzce

University, Düzce, Turkey

\section{Springer}

\begin{abstract}
Simpson inequalities for differentiable convex functions and their fractional versions have been studied extensively. Simpson type inequalities for twice differentiable functions are also investigated. More precisely, Budak et al. established the first result on fractional Simpson inequality for twice differentiable functions. In the present article, we prove a new identity for twice differentiable functions. In addition to this, we establish several fractional Simpson type inequalities for functions whose second derivatives in absolute value are convex. This paper is a new version of fractional Simpson type inequalities for twice differentiable functions.
\end{abstract}

MSC: 26D07; 26D10; 26D15

Keywords: Simpson type inequalities; Convex function; Fractional integrals

\section{Introduction}

Simpson's inequality plays a considerable role in several branches of mathematics. For four times continuously differentiable functions, the classical Simpson's inequality is expressed as follows.

Theorem 1 Suppose that $\mathcal{F}:\left[\rho_{1}, \rho_{2}\right] \rightarrow \mathbb{R}$ is a four times continuously differentiable mapping on $\left(\rho_{1}, \rho_{2}\right)$, and let $\left\|\mathcal{F}^{(4)}\right\|_{\infty}=\sup _{\kappa \in\left(\rho_{1}, \rho_{2}\right)}\left|\mathcal{F}^{(4)}(\kappa)\right|<\infty$. Then one has the inequality

$$
\begin{aligned}
& \left|\frac{1}{3}\left[\frac{\mathcal{F}\left(\rho_{1}\right)+\mathcal{F}\left(\rho_{2}\right)}{2}+2 \mathcal{F}\left(\frac{\rho_{1}+\rho_{2}}{2}\right)\right]-\frac{1}{\rho_{2}-\rho_{1}} \int_{\rho_{1}}^{\rho_{2}} \mathcal{F}(\kappa) d \kappa\right| \\
& \quad \leq \frac{1}{2880}\left\|\mathcal{F}^{(4)}\right\|_{\infty}\left(\rho_{2}-\rho_{1}\right)^{4} .
\end{aligned}
$$

Since the convex theory is an effective way to solve a large number of problems from different branches of mathematics, many authors have studied the results of Simpson type for convex mapping. To be more precise, some inequalities of Simpson type for $s$-convex functions are proved by using differentiable functions [4]. In the papers [34, 36], the new variants of Simpson type inequalities are established based on differentiable convex mapping. Moreover, some papers were devoted to Simpson type inequalities for various convex classes $[11,18,27,30,31]$.

(c) The Author(s) 2021. This article is licensed under a Creative Commons Attribution 4.0 International License, which permits use, sharing, adaptation, distribution and reproduction in any medium or format, as long as you give appropriate credit to the original author(s) and the source, provide a link to the Creative Commons licence, and indicate if changes were made. The images or other third party material in this article are included in the article's Creative Commons licence, unless indicated otherwise in a credit line to the material. If material is not included in the article's Creative Commons licence and your intended use is not permitted by statutory regulation or exceeds the permitted use, you will need to obtain permission directly from the copyright holder. To view a copy of this licence, visit http://creativecommons.org/licenses/by/4.0/. 
The Simpson inequalities for differentiable functions are extended to Riemann-Liouville fractional integrals in the papers [8] and [17]. Hence, several paper focused on fractional Simpson inequalities for various fractional integral operators $[1-3,7,9,12,15,19,21,25$, $26,28,32,33,37,39]$. For further information and unexplained subjects about Simpson type inequalities, we refer the reader to $[5,10,14,16,22-24,38]$ and the references therein. Besides, Sarikaya et al. established several Simpson type inequalities for functions whose second derivatives are convex [35].

The purpose of this paper is to extend the results given in [35] for twice differentiable functions to Riemann-Liouville fractional integrals. The general structure of the paper consists of four chapters including an introduction. The remaining part of the paper proceeds as follows: In Sect. 2, after giving a general literature survey and the definition of Riemann-Liouville fractional integral operators, we prove an equality for twice differentiable functions. In the next section, for utilizing this equality, we establish several Simpson type inequalities for a mapping whose second derivatives are convex. In the last section, some conclusions and further directions of research are discussed.

Definition 1 Consider $\mathcal{F} \in L_{1}\left[\rho_{1}, \rho_{2}\right]$. The Riemann-Liouville integrals $J_{\rho_{1}+}^{\alpha} \mathcal{F}$ and $J_{\rho_{2}-}^{\alpha} \mathcal{F}$ of order $\alpha>0$ with $\rho_{1} \geq 0$ are defined by

$$
J_{\rho_{1}+}^{\alpha} \mathcal{F}(\kappa)=\frac{1}{\Gamma(\alpha)} \int_{\rho_{1}}^{\kappa}(\kappa-\tau)^{\alpha-1} \mathcal{F}(\tau) d \tau, \quad \kappa>\rho_{1}
$$

and

$$
J_{\rho_{2}-}^{\alpha} \mathcal{F}(\kappa)=\frac{1}{\Gamma(\alpha)} \int_{\kappa}^{\rho_{2}}(\tau-\kappa)^{\alpha-1} \mathcal{F}(\tau) d \tau, \quad \kappa<\rho_{2},
$$

respectively. Here, $\Gamma(\alpha)$ is the gamma function and $J_{\rho_{1}+}^{0} \mathcal{F}(\kappa)=J_{\rho_{2}-}^{0} \mathcal{F}(\kappa)=\mathcal{F}(\kappa)$.

For more information and several properties of Riemann-Liouville fractional integrals, please refer to [13, 20, 29].

The first result on fractional Simpson inequality for twice differentiable functions was proved by Budak et al. in [6] as follows.

Theorem 2 Assume that the assumptions of Lemma 1 hold. Assume also that the mapping $\left|\mathcal{F}^{\prime \prime}\right|$ is convex on $\left[\rho_{1}, \rho_{2}\right]$. Then we have the following inequality:

$$
\begin{aligned}
\mid \frac{1}{6}[ & \left.\mathcal{F}\left(\rho_{1}\right)+4 \mathcal{F}\left(\frac{\rho_{1}+\rho_{2}}{2}\right)+\mathcal{F}\left(\rho_{2}\right)\right] \\
& \quad-\frac{2^{\alpha-1} \Gamma(\alpha+1)}{\left(\rho_{2}-\rho_{1}\right)^{\alpha}}\left[J_{\left(\frac{\rho_{1}+\rho_{2}}{2}\right)+}^{\alpha} \mathcal{F}\left(\rho_{2}\right)+J_{\left(\frac{\rho_{1}+\rho_{2}}{2}\right)-}^{\alpha} \mathcal{F}\left(\rho_{1}\right)\right] \mid \\
\quad \leq & \frac{\left(\rho_{2}-\rho_{1}\right)^{2}}{6} \mathcal{A}(\alpha)\left[\left|\mathcal{F}^{\prime \prime}\left(\rho_{1}\right)\right|+\left|\mathcal{F}^{\prime \prime}\left(\rho_{2}\right)\right|\right],
\end{aligned}
$$

where

$$
\mathcal{A}(\alpha)=\frac{1}{4(\alpha+2)}\left(\alpha\left(\frac{\alpha+1}{3}\right)^{\frac{2}{\alpha}}+\frac{3}{\alpha+1}\right)-\frac{1}{8} .
$$

In this paper, we prove a new version of inequality (1.1). 


\section{Some equalities}

In this section, we give equalities on twice differentiable functions for using the main results.

Lemma 1 If $\mathcal{F}:\left[\rho_{1}, \rho_{2}\right] \rightarrow \mathbb{R}$ is an absolutely continuous mapping $\left(\rho_{1}, \rho_{2}\right)$ such that $\mathcal{F}^{\prime \prime} \in$ $L_{1}\left(\left[\rho_{1}, \rho_{2}\right]\right)$, then the following equality

$$
\begin{aligned}
& \frac{1}{6}\left[\mathcal{F}\left(\rho_{1}\right)+4 \mathcal{F}\left(\frac{\rho_{1}+\rho_{2}}{2}\right)+\mathcal{F}\left(\rho_{2}\right)\right] \\
& \quad-\frac{2^{\alpha-1} \Gamma(\alpha+1)}{\left(\rho_{2}-\rho_{1}\right)^{\alpha}}\left[J_{\rho_{2}-}^{\alpha} \mathcal{F}\left(\frac{\rho_{1}+\rho_{2}}{2}\right)+J_{\rho_{1}+}^{\alpha} \mathcal{F}\left(\frac{\rho_{1}+\rho_{2}}{2}\right)\right] \\
& =\frac{\left(\rho_{2}-\rho_{1}\right)^{2}}{8(\alpha+1)} \int_{0}^{1}\left(\frac{1-2 \alpha}{3}+\frac{2(\alpha+1)}{3} \tau-\tau^{\alpha+1}\right) \\
& \quad \times\left[\mathcal{F}^{\prime \prime}\left(\frac{1+\tau}{2} \rho_{2}+\frac{1-\tau}{2} \rho_{1}\right)+\mathcal{F}^{\prime \prime}\left(\frac{1+\tau}{2} \rho_{1}+\frac{1-\tau}{2} \rho_{2}\right)\right] d \tau
\end{aligned}
$$

is valid.

Proof By using integration by parts, we obtain

$$
\begin{aligned}
\Upsilon_{1}= & \int_{0}^{1}\left(\frac{1-2 \alpha}{3}+\frac{2(\alpha+1)}{3} \tau-\tau^{\alpha+1}\right) \mathcal{F}^{\prime \prime}\left(\frac{1+\tau}{2} \rho_{2}+\frac{1-\tau}{2} \rho_{1}\right) d \tau \\
= & -2 \frac{(1-2 \alpha)}{3\left(\rho_{2}-\rho_{1}\right)} \mathcal{F}^{\prime}\left(\frac{\rho_{1}+\rho_{2}}{2}\right) \\
& -\frac{2}{\left(\rho_{2}-\rho_{1}\right)} \int_{0}^{1}\left(\frac{2(\alpha+1)}{3}-(\alpha+1) \tau^{\alpha}\right) \mathcal{F}^{\prime}\left(\frac{1+\tau}{2} \rho_{2}+\frac{1-\tau}{2} \rho_{1}\right) d \tau \\
= & -2 \frac{(1-2 \alpha)}{3\left(\rho_{2}-\rho_{1}\right)} \mathcal{F}^{\prime}\left(\frac{\rho_{1}+\rho_{2}}{2}\right)+\frac{4(\alpha+1)}{3\left(\rho_{2}-\rho_{1}\right)^{2}} \mathcal{F}\left(\rho_{2}\right)+\frac{8(\alpha+1)}{3\left(\rho_{2}-\rho_{1}\right)^{2}} \mathcal{F}\left(\frac{\rho_{1}+\rho_{2}}{2}\right) \\
& -\frac{4 \alpha(\alpha+1)}{\rho_{2}-\rho_{1}} \int_{0}^{1} \tau^{\alpha-1} \mathcal{F}\left(\frac{1+\tau}{2} \rho_{2}+\frac{1-\tau}{2} \rho_{1}\right) d \tau .
\end{aligned}
$$

By using equation (2.2), the change of the variable $\kappa=\frac{1+\tau}{2} \rho_{2}+\frac{1-\tau}{2} \rho_{1}$ for $\tau \in[0,1]$ can be rewritten as follows:

$$
\begin{aligned}
\Upsilon_{1}= & -2 \frac{(1-2 \alpha)}{3\left(\rho_{2}-\rho_{1}\right)} \mathcal{F}^{\prime}\left(\frac{\rho_{1}+\rho_{2}}{2}\right)+\frac{4(\alpha+1)}{3\left(\rho_{2}-\rho_{1}\right)^{2}} \mathcal{F}\left(\rho_{2}\right)+\frac{8(\alpha+1)}{3\left(\rho_{2}-\rho_{1}\right)^{2}} \mathcal{F}\left(\frac{\rho_{1}+\rho_{2}}{2}\right) \\
& -\frac{2^{\alpha+2}(\alpha+1) \Gamma(\alpha+1)}{\left(\rho_{2}-\rho_{1}\right)^{\alpha+2}} J_{\rho_{2}-}^{\alpha} \mathcal{F}\left(\frac{\rho_{1}+\rho_{2}}{2}\right) .
\end{aligned}
$$

Similarly, we have

$$
\begin{aligned}
\Upsilon_{2}= & \int_{0}^{1}\left(\frac{1-2 \alpha}{3}+\frac{2(\alpha+1)}{3} \tau-\tau^{\alpha+1}\right) \mathcal{F}^{\prime \prime}\left(\frac{1+\tau}{2} \rho_{1}+\frac{1-\tau}{2} \rho_{2}\right) d \tau \\
= & 2 \frac{(1-2 \alpha)}{3\left(\rho_{2}-\rho_{1}\right)} \mathcal{F}^{\prime}\left(\frac{\rho_{1}+\rho_{2}}{2}\right)+\frac{4(\alpha+1)}{3\left(\rho_{2}-\rho_{1}\right)^{2}} \mathcal{F}\left(\rho_{1}\right)+\frac{8(\alpha+1)}{3\left(\rho_{2}-\rho_{1}\right)^{2}} \mathcal{F}\left(\frac{\rho_{1}+\rho_{2}}{2}\right) \\
& -\frac{2^{\alpha+2}(\alpha+1) \Gamma(\alpha+1)}{\left(\rho_{2}-\rho_{1}\right)^{\alpha+2}} J_{\rho_{1}+}^{\alpha} \mathcal{F}\left(\frac{\rho_{1}+\rho_{2}}{2}\right) .
\end{aligned}
$$


From equations (2.3) and (2.4), we get

$$
\begin{aligned}
\Upsilon_{1}+\Upsilon_{2}= & \frac{4(\alpha+1)}{3\left(\rho_{2}-\rho_{1}\right)^{2}}\left[\mathcal{F}\left(\rho_{1}\right)+4 \mathcal{F}\left(\frac{\rho_{1}+\rho_{2}}{2}\right)+\mathcal{F}\left(\rho_{2}\right)\right] \\
& -\frac{2^{\alpha+2}(\alpha+1) \Gamma(\alpha+1)}{\left(\rho_{2}-\rho_{1}\right)^{\alpha+2}}\left[J_{\rho_{2}-}^{\alpha} \mathcal{F}\left(\frac{\rho_{1}+\rho_{2}}{2}\right)+J_{\rho_{1+}}^{\alpha} \mathcal{F}\left(\frac{\rho_{1}+\rho_{2}}{2}\right)\right] .
\end{aligned}
$$

Multiplying both sides of (2.5) by $\frac{\left(\rho_{2}-\rho_{1}\right)^{2}}{8(\alpha+1)}$, we obtain equation (2.1). This ends the proof of Lemma 1.

Lemma 2 Let us consider the function $\varpi:[0,1] \rightarrow \mathbb{R}$ by $\varpi(\tau)=\frac{1-2 \alpha}{3}+\frac{2(\alpha+1)}{3} \tau-\tau^{\alpha+1}$ with $\alpha>0$.

(1) If $0<\alpha \leq \frac{1}{2}$, then we have

$$
\int_{0}^{1}|\varpi(\tau)| d \tau=\frac{1-\alpha^{2}}{3(\alpha+2)}
$$

(2) If $\alpha>\frac{1}{2}$, then there exists a real number $\varsigma_{\alpha}$ such that $0<\varsigma_{\alpha}<1$, and we have

$$
\int_{0}^{1}|\varpi(\tau)| d \tau=2\left(\frac{\left(\varsigma_{\alpha}\right)^{\alpha+2}}{\alpha+2}-\frac{(1-2 \alpha) \varsigma_{\alpha}+(\alpha+1)\left(\varsigma_{\alpha}\right)^{2}}{3}\right)+\frac{1-\alpha^{2}}{3(\alpha+2)} .
$$

Proof Let us note that $0<\alpha \leq \frac{1}{2}$. Then $\varpi(\tau) \geq 0$ for all $\tau \in[0,1]$. Thus, it can be easily seen that

$$
\int_{0}^{1}|\varpi(\tau)| d \tau=\int_{0}^{1} \varpi(\tau) d \tau=\frac{1-\alpha^{2}}{3(\alpha+2)} .
$$

If $\alpha>\frac{1}{2}$, then there exists a real number $\varsigma_{\alpha} \in(0,1)$ such that $\varpi(\tau) \leq 0$ for $0 \leq \tau \leq \varsigma_{\alpha}$ and $\varpi(\tau) \geq 0$ for $\varsigma_{\alpha} \leq \tau \leq 1$. Therefore, we obtain

$$
\begin{aligned}
\int_{0}^{1}|\varpi(\tau)| d \tau & =\int_{0}^{\zeta \alpha}(-\varpi(\tau)) d \tau+\int_{\varsigma_{\alpha}}^{1} \varpi(\tau) d \tau \\
& =2\left(\frac{\left(\zeta_{\alpha}\right)^{\alpha+2}}{\alpha+2}-\frac{(1-2 \alpha) \zeta_{\alpha}+(\alpha+1)\left(\varsigma_{\alpha}\right)^{2}}{3}\right)+\frac{1-\alpha^{2}}{3(\alpha+2)}
\end{aligned}
$$

Lemma 3 Define the function $\varpi:[0,1] \rightarrow \mathbb{R}$ by $\varpi(\tau)=\frac{1-2 \alpha}{3}+\frac{2(\alpha+1)}{3} \tau-\tau^{\alpha+1}$ with $\alpha>0$.

(1) Let us consider $0<\alpha \leq \frac{1}{2}$. Then we have

$$
\int_{0}^{1}|\varpi(\tau)| \tau d \tau=\frac{3-\alpha-2 \alpha^{2}}{18(\alpha+3)}
$$

(2) If we take $\alpha>\frac{1}{2}$, then there exists a real number $\varsigma_{\alpha}$ so that $0<\varsigma_{\alpha}<1$, and we get

$$
\int_{0}^{1}|\varpi(\tau)| \tau d \tau=2\left(\frac{\left(\varsigma_{\alpha}\right)^{\alpha+3}}{\alpha+3}-\frac{3(1-2 \alpha)\left(\varsigma_{\alpha}\right)^{2}+4(\alpha+1)\left(\varsigma_{\alpha}\right)^{3}}{18}\right)+\frac{3+\alpha-2 \alpha^{2}}{18(\alpha+3)}
$$

Proof The proof can be done similar to the proof of Lemma 2. 


\section{New Simpson type inequalities for twice differentiable functions}

In this section, we prove several Simpson type inequalities for a mapping whose second derivatives are convex.

Theorem 3 Let us note that the assumptions of Lemma 1 are valid. Let us also note that the mapping $\left|\mathcal{F}^{\prime \prime}\right|$ is convex on $\left[\rho_{1}, \rho_{2}\right]$. Then we have the following inequality:

$$
\begin{aligned}
\mid \frac{1}{6}[ & \left.\mathcal{F}\left(\rho_{1}\right)+4 \mathcal{F}\left(\frac{\rho_{1}+\rho_{2}}{2}\right)+\mathcal{F}\left(\rho_{2}\right)\right] \\
& \quad-\frac{2^{\alpha-1} \Gamma(\alpha+1)}{\left(\rho_{2}-\rho_{1}\right)^{\alpha}}\left[J_{\rho_{2}}^{\alpha} \mathcal{F}\left(\frac{\rho_{1}+\rho_{2}}{2}\right)+J_{\rho_{1}+}^{\alpha} \mathcal{F}\left(\frac{\rho_{1}+\rho_{2}}{2}\right)\right] \mid \\
& \leq \frac{\left(\rho_{2}-\rho_{1}\right)^{2}}{8(\alpha+1)} \Omega_{1}(\alpha)\left[\left|\mathcal{F}^{\prime \prime}\left(\rho_{1}\right)\right|+\left|\mathcal{F}^{\prime \prime}\left(\rho_{2}\right)\right|\right],
\end{aligned}
$$

where $\Omega_{1}$ is defined by

$$
\Omega_{1}(\alpha)= \begin{cases}\frac{1-\alpha^{2}}{3(\alpha+2)}, & \text { if } 0<\alpha \leq \frac{1}{2} \\ 2\left(\frac{(5 \alpha)^{\alpha+2}}{\alpha+2}-\frac{(1-2 \alpha)_{5 \alpha}+(\alpha+1)\left(\zeta_{\alpha}\right)^{2}}{3}\right)+\frac{1-\alpha^{2}}{3(\alpha+2)}, & \text { if } \alpha>\frac{1}{2} .\end{cases}
$$

Proof By taking modulus in Lemma 1, we have

$$
\begin{aligned}
& \mid \frac{1}{6}[\left.\mathcal{F}\left(\rho_{1}\right)+4 \mathcal{F}\left(\frac{\rho_{1}+\rho_{2}}{2}\right)+\mathcal{F}\left(\rho_{2}\right)\right] \\
&-\frac{2^{\alpha-1} \Gamma(\alpha+1)}{\left(\rho_{2}-\rho_{1}\right)^{\alpha}}\left[J_{\rho_{2}-}^{\alpha} \mathcal{F}\left(\frac{\rho_{1}+\rho_{2}}{2}\right)+J_{\rho_{1}+}^{\alpha} \mathcal{F}\left(\frac{\rho_{1}+\rho_{2}}{2}\right)\right] \mid \\
& \leq \frac{\left(\rho_{2}-\rho_{1}\right)^{2}}{8(\alpha+1)} \int_{0}^{1}\left|\frac{1-2 \alpha}{3}+\frac{2(\alpha+1)}{3} \tau-\tau^{\alpha+1}\right| d \tau \\
& \quad \times\left[\left|\mathcal{F}^{\prime \prime}\left(\frac{1+\tau}{2} \rho_{2}+\frac{1-\tau}{2} \rho_{1}\right)\right|+\left|\mathcal{F}^{\prime \prime}\left(\frac{1+\tau}{2} \rho_{1}+\frac{1-\tau}{2} \rho_{2}\right)\right|\right] d \tau .
\end{aligned}
$$

By using the convexity of $\left|\mathcal{F}^{\prime \prime}\right|$, we obtain

$$
\begin{aligned}
& \mid \frac{1}{6}\left[\mathcal{F}\left(\rho_{1}\right)+4 \mathcal{F}\left(\frac{\rho_{1}+\rho_{2}}{2}\right)+\mathcal{F}\left(\rho_{2}\right)\right] \\
& \quad-\frac{2^{\alpha-1} \Gamma(\alpha+1)}{\left(\rho_{2}-\rho_{1}\right)^{\alpha}}\left[J_{\rho_{2}-}^{\alpha} \mathcal{F}\left(\frac{\rho_{1}+\rho_{2}}{2}\right)+J_{\rho_{1}+}^{\alpha} \mathcal{F}\left(\frac{\rho_{1}+\rho_{2}}{2}\right)\right] \mid \\
& \leq \frac{\left(\rho_{2}-\rho_{1}\right)^{2}}{8(\alpha+1)}\left[\int_{0}^{1}\left|\frac{1-2 \alpha}{3}+\frac{2(\alpha+1)}{3} \tau-\tau^{\alpha+1}\right|\right. \\
& \quad \times\left[\left(\frac{1+\tau}{2}\right)\left|\mathcal{F}^{\prime \prime}\left(\rho_{2}\right)\right|+\left(\frac{1-\tau}{2}\right)\left|\mathcal{F}^{\prime \prime}\left(\rho_{1}\right)\right|\right] d \tau \\
& \left.\quad+\left(\frac{1+\tau}{2}\right)\left|\mathcal{F}^{\prime \prime}\left(\rho_{1}\right)\right|+\left(\frac{1-\tau}{2}\right)\left|\mathcal{F}^{\prime \prime}\left(\rho_{2}\right)\right|\right] d \tau
\end{aligned}
$$




$$
\begin{aligned}
& =\frac{\left(\rho_{2}-\rho_{1}\right)^{2}}{8(\alpha+1)} \int_{0}^{1}\left|\frac{1-2 \alpha}{3}+\frac{2(\alpha+1)}{3} \tau-\tau^{\alpha+1}\right| d \tau\left[\left|\mathcal{F}^{\prime \prime}\left(\rho_{2}\right)\right|+\left|\mathcal{F}^{\prime \prime}\left(\rho_{1}\right)\right|\right] \\
& =\frac{\left(\rho_{2}-\rho_{1}\right)^{2}}{8(\alpha+1)} \Omega_{1}(\alpha)\left[\left|\mathcal{F}^{\prime \prime}\left(\rho_{1}\right)\right|+\left|\mathcal{F}^{\prime \prime}\left(\rho_{2}\right)\right|\right] .
\end{aligned}
$$

This completes the proof of Theorem 3.

Theorem 4 Let us consider that the assumptions of Lemma 1 hold. If the mapping $\left|\mathcal{F}^{\prime \prime}\right|^{q}$, $q>1$ is convex on $\left[\rho_{1}, \rho_{2}\right]$, then we have the following inequality:

$$
\begin{aligned}
\mid \frac{1}{6} & {\left[\mathcal{F}\left(\rho_{1}\right)+4 \mathcal{F}\left(\frac{\rho_{1}+\rho_{2}}{2}\right)+\mathcal{F}\left(\rho_{2}\right)\right] } \\
& \quad-\frac{2^{\alpha-1} \Gamma(\alpha+1)}{\left(\rho_{2}-\rho_{1}\right)^{\alpha}}\left[J_{\rho_{2}-}^{\alpha} \mathcal{F}\left(\frac{\rho_{1}+\rho_{2}}{2}\right)+J_{\rho_{1}+}^{\alpha} \mathcal{F}\left(\frac{\rho_{1}+\rho_{2}}{2}\right)\right] \mid \\
\leq & \frac{\left(\rho_{2}-\rho_{1}\right)^{2}}{8(\alpha+1)} \Psi(\alpha, p)\left[\left|\mathcal{F}^{\prime \prime}\left(\rho_{1}\right)\right|^{q}+\left|\mathcal{F}^{\prime \prime}\left(\rho_{2}\right)\right|^{q}\right]^{\frac{1}{q}} .
\end{aligned}
$$

Here, $\frac{1}{p}+\frac{1}{q}=1$ and $\Psi$ is defined by

$$
\Psi(\alpha, p)=\left(\int_{0}^{1}\left|\frac{1-2 \alpha}{3}+\frac{2(\alpha+1)}{3} \tau-\tau^{\alpha+1}\right|^{p} d \tau\right)^{\frac{1}{p}} .
$$

Proof With the help of Hölder's inequality in inequality (3.1), we get

$$
\begin{aligned}
& \mid \frac{1}{6}\left[\mathcal{F}\left(\rho_{1}\right)+4 \mathcal{F}\left(\frac{\rho_{1}+\rho_{2}}{2}\right)+\mathcal{F}\left(\rho_{2}\right)\right] \\
& \quad-\frac{2^{\alpha-1} \Gamma(\alpha+1)}{\left(\rho_{2}-\rho_{1}\right)^{\alpha}}\left[J_{\rho_{2}-}^{\alpha} \mathcal{F}\left(\frac{\rho_{1}+\rho_{2}}{2}\right)+J_{\rho_{1}+}^{\alpha} \mathcal{F}\left(\frac{\rho_{1}+\rho_{2}}{2}\right)\right] \mid \\
& \leq \frac{\left(\rho_{2}-\rho_{1}\right)^{2}}{8(\alpha+1)}\left\{\left(\left.\int_{0}^{1}\left|\frac{1-2 \alpha}{3}+\frac{2(\alpha+1)}{3} \tau-\tau^{\alpha+1}\right|\right|^{p} d \tau\right)^{\frac{1}{p}}\right. \\
& \quad \times\left(\int_{0}^{1}\left|\mathcal{F}^{\prime \prime}\left(\frac{1+\tau}{2} \rho_{2}+\frac{1-\tau}{2} \rho_{1}\right)\right|^{q} d \tau\right)^{\frac{1}{q}} \\
& \quad+\left(\int_{0}^{1}\left|\frac{1-2 \alpha}{3}+\frac{2(\alpha+1)}{3} \tau-\tau^{\alpha+1}\right|^{p} d \tau\right)^{\frac{1}{p}} \\
& \left.\quad \times\left(\int_{0}^{1}\left|\mathcal{F}^{\prime \prime}\left(\frac{1+\tau}{2} \rho_{1}+\frac{1-\tau}{2} \rho_{2}\right)\right|^{q} d \tau\right)^{\frac{1}{q}}\right\} .
\end{aligned}
$$

By using the convexity of $\left|\mathcal{F}^{\prime \prime}\right|^{q}$, we obtain

$$
\begin{aligned}
& \mid \frac{1}{6}\left[\mathcal{F}\left(\rho_{1}\right)+4 \mathcal{F}\left(\frac{\rho_{1}+\rho_{2}}{2}\right)+\mathcal{F}\left(\rho_{2}\right)\right] \\
& \quad-\frac{2^{\alpha-1} \Gamma(\alpha+1)}{\left(\rho_{2}-\rho_{1}\right)^{\alpha}}\left[J_{\rho_{2}-}^{\alpha} \mathcal{F}\left(\frac{\rho_{1}+\rho_{2}}{2}\right)+J_{\rho_{1}+}^{\alpha} \mathcal{F}\left(\frac{\rho_{1}+\rho_{2}}{2}\right)\right] \mid \\
& \quad \leq \frac{\left(\rho_{2}-\rho_{1}\right)^{2}}{8(\alpha+1)}\left(\int_{0}^{1}\left|\frac{1-2 \alpha}{3}+\frac{2(\alpha+1)}{3} \tau-\tau^{\alpha+1}\right|^{p} d \tau\right)^{\frac{1}{p}}
\end{aligned}
$$




$$
\begin{aligned}
& \times\left[\left(\int_{0}^{1}\left[\left(\frac{1+\tau}{2}\right)\left|\mathcal{F}^{\prime \prime}\left(\rho_{2}\right)\right|^{q}+\left(\frac{1-\tau}{2}\right)\left|\mathcal{F}^{\prime \prime}\left(\rho_{1}\right)\right|^{q}\right] d \tau\right)^{\frac{1}{q}}\right. \\
& \left.+\left(\int_{0}^{1}\left[\left(\frac{1+\tau}{2}\right)\left|\mathcal{F}^{\prime \prime}\left(\rho_{1}\right)\right|^{q}+\left(\frac{1-\tau}{2}\right)\left|\mathcal{F}^{\prime \prime}\left(\rho_{2}\right)\right|^{q}\right] d \tau\right)^{\frac{1}{q}}\right] \\
= & \frac{\left(\rho_{2}-\rho_{1}\right)^{2}}{8(\alpha+1)}\left(\int_{0}^{1}\left|\frac{1-2 \alpha}{3}+\frac{2(\alpha+1)}{3} \tau-\tau^{\alpha+1}\right|^{p} d \tau\right)^{\frac{1}{p}} \\
& \times\left[\left(\frac{3\left|\mathcal{F}^{\prime \prime}\left(\rho_{2}\right)\right|^{q}+\left|\mathcal{F}^{\prime \prime}\left(\rho_{1}\right)\right|^{q}}{4}\right)^{\frac{1}{q}}+\left(\frac{\left|\mathcal{F}^{\prime \prime}\left(\rho_{2}\right)\right|^{q}+3\left|\mathcal{F}^{\prime \prime}\left(\rho_{1}\right)\right|^{q}}{4}\right)^{\frac{1}{q}}\right] .
\end{aligned}
$$

This finishes the proof of Theorem 4.

Theorem 5 Suppose that the assumptions of Lemma 1 hold. If the mapping $\left|\mathcal{F}^{\prime \prime}\right|^{q}, q \geq 1$ is convex on $\left[\rho_{1}, \rho_{2}\right]$, then we have the following inequality:

$$
\begin{aligned}
& \mid \frac{1}{6}[\left.\mathcal{F}\left(\rho_{1}\right)+4 \mathcal{F}\left(\frac{\rho_{1}+\rho_{2}}{2}\right)+\mathcal{F}\left(\rho_{2}\right)\right] \\
& \quad-\frac{2^{\alpha-1} \Gamma(\alpha+1)}{\left(\rho_{2}-\rho_{1}\right)^{\alpha}}\left[J_{\rho_{2}-}^{\alpha} \mathcal{F}\left(\frac{\rho_{1}+\rho_{2}}{2}\right)+J_{\rho_{1}+}^{\alpha} \mathcal{F}\left(\frac{\rho_{1}+\rho_{2}}{2}\right)\right] \mid \\
& \leq \frac{\left(\rho_{2}-\rho_{1}\right)^{2}}{8(\alpha+1)}\left(\Omega_{1}(\alpha)\right)^{1-\frac{1}{q}} \\
& \quad \times\left\{\left(\frac{\left(\Omega_{1}(\alpha)+\Omega_{2}(\alpha)\right)\left|\mathcal{F}^{\prime \prime}\left(\rho_{2}\right)\right|^{q}+\left(\Omega_{1}(\alpha)-\Omega_{2}(\alpha)\right)\left|\mathcal{F}^{\prime \prime}\left(\rho_{1}\right)\right|^{q}}{2}\right)^{\frac{1}{q}}\right. \\
&\left.\quad+\left(\frac{\left(\Omega_{1}(\alpha)+\Omega_{2}(\alpha)\right)\left|\mathcal{F}^{\prime \prime}\left(\rho_{1}\right)\right|^{q}+\left(\Omega_{1}(\alpha)-\Omega_{2}(\alpha)\right)\left|\mathcal{F}^{\prime \prime}\left(\rho_{2}\right)\right|^{q}}{2}\right)^{\frac{1}{q}}\right\},
\end{aligned}
$$

where $\Omega_{1}$ is defined as in Theorem 3 and $\Omega_{2}$ is defined by

$$
\Omega_{2}(\alpha)= \begin{cases}\frac{3-\alpha-2 \alpha^{2}}{18(\alpha+3)}, & \text { if } 0<\alpha \leq \frac{1}{2}, \\ 2\left(\frac{(5 \alpha)^{\alpha+3}}{\alpha+3}-\frac{3(1-2 \alpha)(\varsigma \alpha)^{2}+4(\alpha+1)(\varsigma \alpha)^{3}}{18}\right)+\frac{3+\alpha-2 \alpha^{2}}{18(\alpha+3)}, & \text { if } \alpha>\frac{1}{2} .\end{cases}
$$

Proof By applying the power-mean inequality in (3.1), we get

$$
\begin{aligned}
\mid \frac{1}{6}[ & \left.\mathcal{F}\left(\rho_{1}\right)+4 \mathcal{F}\left(\frac{\rho_{1}+\rho_{2}}{2}\right)+\mathcal{F}\left(\rho_{2}\right)\right] \\
& -\frac{2^{\alpha-1} \Gamma(\alpha+1)}{\left(\rho_{2}-\rho_{1}\right)^{\alpha}}\left[J_{\rho_{2}-}^{\alpha} \mathcal{F}\left(\frac{\rho_{1}+\rho_{2}}{2}\right)+J_{\rho_{1}+}^{\alpha} \mathcal{F}\left(\frac{\rho_{1}+\rho_{2}}{2}\right)\right] \mid \\
\leq & \frac{\left(\rho_{2}-\rho_{1}\right)^{2}}{8(\alpha+1)}\left[\left(\int_{0}^{1}\left|\frac{1-2 \alpha}{3}+\frac{2(\alpha+1)}{3} \tau-\tau^{\alpha+1}\right| d \tau\right)^{1-\frac{1}{q}}\right. \\
& \times\left(\int_{0}^{1}\left|\frac{1-2 \alpha}{3}+\frac{2(\alpha+1)}{3} \tau-\tau^{\alpha+1}\right|\left|\mathcal{F}^{\prime \prime}\left(\frac{1+\tau}{2} \rho_{2}+\frac{1-\tau}{2} \rho_{1}\right)\right|^{q} d \tau\right)^{\frac{1}{q}} \\
& +\left(\int_{0}^{1}\left|\frac{1-2 \alpha}{3}+\frac{2(\alpha+1)}{3} \tau-\tau^{\alpha+1}\right| d \tau\right)^{1-\frac{1}{q}} \\
& \left.\times\left(\int_{0}^{1}\left|\frac{1-2 \alpha}{3}+\frac{2(\alpha+1)}{3} \tau-\tau^{\alpha+1}\right|\left|\mathcal{F}^{\prime \prime}\left(\frac{1+\tau}{2} \rho_{1}+\frac{1-\tau}{2} \rho_{2}\right)\right|^{q} d \tau\right)^{\frac{1}{q}}\right] .
\end{aligned}
$$


Since $\left|\mathcal{F}^{\prime \prime}\right|^{q}$ is convex, we obtain

$$
\begin{aligned}
& \int_{0}^{1}\left|\frac{1-2 \alpha}{3}+\frac{2(\alpha+1)}{3} \tau-\tau^{\alpha+1}\right|\left|\mathcal{F}^{\prime \prime}\left(\frac{1+\tau}{2} \rho_{2}+\frac{1-\tau}{2} \rho_{1}\right)\right|^{q} d \tau \\
& \quad \leq \int_{0}^{1}\left|\frac{1-2 \alpha}{3}+\frac{2(\alpha+1)}{3} \tau-\tau^{\alpha+1}\right|\left[\frac{1+\tau}{2}\left|\mathcal{F}^{\prime \prime}\left(\rho_{2}\right)\right|^{q}+\frac{1-\tau}{2}\left|\mathcal{F}^{\prime \prime}\left(\rho_{1}\right)\right|^{q}\right] d \tau \\
& \quad=\frac{\left(\Omega_{1}(\alpha)+\Omega_{2}(\alpha)\right)\left|\mathcal{F}^{\prime \prime}\left(\rho_{2}\right)\right|^{q}+\left(\Omega_{1}(\alpha)-\Omega_{2}(\alpha)\right)\left|\mathcal{F}^{\prime \prime}\left(\rho_{1}\right)\right|^{q}}{2},
\end{aligned}
$$

and similarly

$$
\begin{gathered}
\int_{0}^{1}\left|\frac{1-2 \alpha}{3}+\frac{2(\alpha+1)}{3} \tau-\tau^{\alpha+1}\right|\left|\mathcal{F}^{\prime \prime}\left(\frac{1+\tau}{2} \rho_{1}+\frac{1-\tau}{2} \rho_{2}\right)\right|^{q} d \tau \\
\leq \frac{\left(\Omega_{1}(\alpha)+\Omega_{2}(\alpha)\right)\left|\mathcal{F}^{\prime \prime}\left(\rho_{1}\right)\right|^{q}+\left(\Omega_{1}(\alpha)-\Omega_{2}(\alpha)\right)\left|\mathcal{F}^{\prime \prime}\left(\rho_{2}\right)\right|^{q}}{2} .
\end{gathered}
$$

Then we obtain the desired result Theorem 5 .

\section{Special cases}

In this section, we present special cases of the main findings in the paper.

Remark 1 If we choose $\alpha=1$ in Theorem 3 , then $\varsigma_{\alpha}=\frac{1}{3}$, and we have the inequality

$$
\begin{aligned}
& \left|\frac{1}{6}\left[\mathcal{F}\left(\rho_{1}\right)+4 \mathcal{F}\left(\frac{\rho_{1}+\rho_{2}}{2}\right)+\mathcal{F}\left(\rho_{2}\right)\right]-\frac{1}{\rho_{2}-\rho_{1}} \int_{\rho_{1}}^{\rho_{2}} \mathcal{F}(\kappa) d \kappa\right| \\
& \quad \leq \frac{\left(\rho_{2}-\rho_{1}\right)^{2}}{162}\left[\left|\mathcal{F}^{\prime \prime}\left(\rho_{1}\right)\right|+\left|\mathcal{F}^{\prime \prime}\left(\rho_{2}\right)\right|\right],
\end{aligned}
$$

which is proved by Sarikaya et al. in [35].

Corollary 1 In Theorem 4, if we assign $\alpha=1$, then $\varsigma_{\alpha}=\frac{1}{3}$, and the following inequality

$$
\begin{aligned}
& \left|\frac{1}{6}\left[\mathcal{F}\left(\rho_{1}\right)+4 \mathcal{F}\left(\frac{\rho_{1}+\rho_{2}}{2}\right)+\mathcal{F}\left(\rho_{2}\right)\right]-\frac{1}{\rho_{2}-\rho_{1}} \int_{\rho_{1}}^{\rho_{2}} \mathcal{F}(\kappa) d \kappa\right| \\
& \quad \leq \frac{\left(\rho_{2}-\rho_{1}\right)^{2}}{16} \Psi(1, p)\left[\left|\mathcal{F}^{\prime \prime}\left(\rho_{1}\right)\right|^{q}+\left|\mathcal{F}^{\prime \prime}\left(\rho_{2}\right)\right|^{q}\right]^{\frac{1}{q}}
\end{aligned}
$$

is valid.

Remark 2 If we take $\alpha=1$ in Theorem 5, then Theorem 5 reduces to [35, Theorem 2.5].

\section{Conclusion}

In the present article, fractional version of Simpson type inequality for twice differentiable functions are established. Moreover, we show that our results generalize the inequalities obtained by Sarikaya et al. [35]. This work is a new version of fractional Simpson type inequalities for twice differentiable functions. In the future studies, authors can try to generalize our results by utilizing a different kind of convex function classes or another type fractional integral operators. In addition to this, the authors can give some applications of special cases with the help of our results. 


\section{Acknowledgements}

The authors would like to express their sincere thanks to the editor and the anonymous reviewers for their helpful comments and suggestions.

Funding

There is no funding.

\section{Availability of data and materials}

Data sharing not applicable to this paper as no data sets were generated or analysed during the current study.

\section{Declarations}

\section{Competing interests}

The authors declare that they have no competing interests.

\section{Authors' contributions}

All authors contributed equally to the writing of this paper. All authors read and approved the final manuscript.

\section{Publisher's Note}

Springer Nature remains neutral with regard to jurisdictional claims in published maps and institutional affiliations.

\section{Received: 28 June 2021 Accepted: 7 September 2021 Published online: 18 October 2021}

\section{References}

1. Abdeljawad, T., Rashid, S., Hammouch, Z., Iscan, I., Chu, Y.M.: Some new Simpson-type inequalities for generalized p-convex function on fractal sets with applications. Adv. Differ. Equ. 2020(1), 496 (2020)

2. Agarwal, P.: Some inequalities involving Hadamard-type $k$-fractional integral operators. Math. Methods Appl. Sci. 40(11), 3882-3891 (2017)

3. Agarwal, P., Jeli, M., Tomar, M.: Certain Hermite-Hadamard type inequalities via generalized $k$-fractional integrals. J. Inequal. Appl. 2017, 55 (2017). https://doi.org/10.1186/s13660-017-1318-y

4. Alomari, M., Darus, M., Dragomir, S.S.: New inequalities of Simpson's type for s-convex functions with applications, RGMIA Res. Rep. Collect. 12(4) (2009)

5. Budak, H., Erden, S., Ali, M.A.: Simpson and Newton type inequalities for convex functions via newly defined quantum integrals. Math. Methods Appl. Sci. 44(1), 378-390 (2021)

6. Budak, H., Kara, H., Hezenci, F.: Fractional Simpson type inequalities for twice differentiable functions (2021, submitted)

7. Chand, M., Prajapati, J.C., Bonyah, E.: Fractional integrals and solution of fractional kinetic equations involving k-Mittag-Leffler function. Trans. A. Razmadze Math. Inst. 171(2), 144-166 (2017)

8. Chen, J., Huang, X.: Some new inequalities of Simpson's type for s-convex functions via fractional integrals. Filomat 31(15), 4989-4997 (2017)

9. Choi, J., Agarwal, P.: Some new Saigo type fractional integral inequalities and their $q$-analogues. Abstr. Appl. Anal. 2014, Article ID 579260 (2014). https://doi.org/10.1155/2014/579260

10. Dragomir, S.S., Agarwal, R.P., Cerone, P.: On Simpson's inequality and applications. J. Inequal. Appl. 5, 533-579 (2000)

11. Du, T., Li, Y., Yang, Z: A generalization of Simpson's inequality via differentiable mapping using extended $(s, m)$-convex functions. Appl. Math. Comput. 293, 358-369 (2017)

12. Ertuğral, F., Sarikaya, M.Z.: Simpson type integral inequalities for generalized fractional integral. Rev. R. Acad. Cienc. Exactas Fís. Nat. Ser. A Mat. 113(4), 3115-3124 (2019)

13. Gorenflo, R., Mainardi, F.: Fractional Calculus: Integral and Differential Equations of Fractional Order, pp. 223-276. Springer, Vienna (1997)

14. Hua, J., Xi, B.Y., Qi, F.: Some new inequalities of Simpson type for strongly s-convex functions. Afr. Math. 26(5), 741-752 (2015)

15. Hussain, S., Khalid, J., Chu, Y.M.: Some generalized fractional integral Simpson's type inequalities with applications AIMS Math. 5(6), 5859-5883 (2020)

16. Hussain, S., Qaisar, S.: More results on Simpson's type inequality through convexity for twice differentiable continuous mappings. SpringerPlus 5(1), 77 (2016)

17. Iqbal, M., Qaisar, S., Hussain, S.: On Simpson's type inequalities utilizing fractional integrals. J. Comput. Anal. Appl. 23(6), 1137-1145 (2017)

18. Isscan, i.: Hermite-Hadamard and Simpson-like type inequalities for differentiable harmonically convex functions. J. Math. 2014, Article ID 346305 (2014)

19. Kermausuor, S.: Simpson's type inequalities via the Katugampola fractional integrals for $s$-convex functions. Kragujev. J. Math. 45(5), 709-720 (2021)

20. Kilbas, A.A., Srivastava, H.M., Trujillo, J.J.: Theory and Applications of Fractional Differential Equations. North-Holland Mathematics Studies, vol. 204. Elsevier, Amsterdam (2006)

21. Lei, H., Hu, G., Nie, J., Du, T.: Generalized Simpson-type inequalities considering first derivatives through the $k$-fractional integrals. IAENG Int. J. Appl. Math. 50(3), 1-8 (2020)

22. $L i, Y$., $\mathrm{Du}, \mathrm{T}$.: Some Simpson type integral inequalities for functions whose third derivatives are $(\alpha, m)$-GA-convex functions. J. Egypt. Math. Soc. 24(2), 175-180 (2016)

23. Liu, B.Z: An inequality of Simpson type. Proc. R. Soc. A 461, 2155-2158 (2005)

24. Liu, W.: Some Simpson type inequalities for $h$-convex and $(\alpha, m)$-convex functions. J. Comput. Anal. Appl. 16(5), 1005-1012 (2014) 
25. Liu, X., Zhang, L., Agarwal, P., Wang, G.: On some new integral inequalities of Gronwall-Bellman-Bihari type with delay for discontinuous functions and their applications. Indag. Math. 27(1), 1-10 (2016)

26. Luo, C., Du, T:: Generalized Simpson type inequalities involving Riemann-Liouville fractional integrals and their applications. Filomat 34(3), 751-760 (2020)

27. Matloka, M.: Some inequalities of Simpson type for $h$-convex functions via fractional integrals. Abstr. Appl. Anal. 2015, Article ID 956850 (2015)

28. Mehrez, K., Agarwal, P.: New Hermite-Hadamard type integral inequalities for convex functions and their applications. J. Comput. Appl. Math. 350, 274-285 (2019)

29. Miller, S., Ross, B.: An Introduction to the Fractional Calculus and Fractional Differential Equations. Wiley, New York (1993)

30. Ozdemir, M.E., Akdemir, A.O., Kavurmaci, H.: On the Simpson's inequality for convex functions on the coordinates. Turk. J. Anal. Number Theory 2(5), 165-169 (2014)

31. Park, J.: On Simpson like type integral inequalities for differentiable preinvex functions. Appl. Math. Sci. 7(121), 6009-6021 (2013)

32. Rashid, S., Akdemir, A.O., Jarad, F., Noor, M.A., Noor, K.I.: Simpson's type integral inequalities for $k$-fractional integrals and their applications. AIMS Math. 4(4), 1087-1100 (2019)

33. Sarikaya, M.Z., Budak, H., Erden, S.: On new inequalities of Simpson's type for generalized convex functions. Korean J. Math. 27(2), 279-295 (2019)

34. Sarikaya, M.Z., Set, E., Ozdemir, M.E.: On new inequalities of Simpson's type for convex functions. RGMIA Res. Rep. Collect. 13(2), Article 2 (2010)

35. Sarikaya, M.Z., Set, E., Ozdemir, M.E.: On new inequalities of Simpson's type for functions whose second derivatives absolute values are convex. J. Appl. Math. Stat. Inform. 9(1), 37-45 (2013)

36. Sarikaya, M.Z., Set, E., Ozdemir, M.E.: On new inequalities of Simpson's type for s-convex functions. Comput. Math. Appl. 60(8), 2191-2199 (2020)

37. Set, E., Akdemir, A.O., Ozdemir, M.E.: Simpson type integral inequalities for convex functions via Riemann-Liouville integrals. Filomat 31(14), 4415-4420 (2017)

38. Vivas-Cortez, M., Abdeljawad, T., Mohammed, P.O., Rangel-Oliveros, Y.: Simpson's integral inequalities for twice differentiable convex functions. Math. Probl. Eng. 2020, Article ID 1936461 (2020)

39. Wang, G., Agarwal, P., Chand, M.: Certain Gruss type inequalities involving the generalized fractional integral operator. J. Inequal. Appl. 2014, 147 (2014). https://doi.org/10.1186/1029-242X-2014-147

\section{Submit your manuscript to a SpringerOpen ${ }^{\circ}$ journal and benefit from:}

- Convenient online submission

- Rigorous peer review

- Open access: articles freely available online

- High visibility within the field

- Retaining the copyright to your article

Submit your next manuscript at $\gg$ springeropen.com 